\title{
Adaptation of the AFLP technique as a new tool to detect genetic instability and transposition in interspecific hybrids
}

\author{
Doris Vela, María Pilar García Guerreiro, and Antonio Fontdevila \\ Grup de Biologia Evolutiva, Departament de Genética i Microbiología, Universitat Autonoma de Barcelona, \\ Bellaterra, Barcelona, Spain
}

BioTechniques 50:247-250 (April 2011) doi 10.2144/000113655

Keywords: Drosophila; interspecific hybrids; AFLP; FISH; transposition; instability markers

An adapted amplified fragment length polymorphism (AFLP) protocol is presented for detection of hybrid instability in the genome of interspecific hybrids between Drosophila buzzatii and D. koepferae species. Analyses of 15 AFLP instability markers (new bands detected in hybrids) show that up to $81 \%$ are the result of transposable element (TE) activity. Twenty TEs associated with AFLP instability markers have been detected by this method in backcross hybrids and segmental hybrids, demonstrating its validity in detecting transposition events occurring during the hybridization process. New insertions of Helena TE have been observed in the hybrid genome after hybridization of the TGTCG22 instability marker by FISH. The AFLP marker technique proved to be an efficient method that improves upon traditional and bioinformatic tools previously used to detect TE mobilization. This newly adapted AFLP protocol may also be applied to a large number of organisms outside the Drosophila genus, making it of interest to evolutionary and population genetic researchers working with species where the knowledge of the genome is scarce.

Hybridization occurs in nature more frequently than formerly expected and is followed by a high level of genetic instability in the form of mutations, chromosomal rearrangements, and transposable element (TE) activation in hybrid genomes (1). Some studies with interspecific hybrids have shown a direct relationship between hybridization and transposition rate increases $(2,3)$. Studies of interspecific hybrids between Drosophila buzzatii and $D$. koepferae species revealed an increase in transposition of the Osvaldo retrotransposon in hybrids when compared with parental species (2).

New experimental techniques and computational tools are being implemented with the objective of obtaining more information about TE mobilization, including an estimation of TE transposition rates. The amplified fragment length polymorphism (AFLP) technique is a DNA fingerprinting tool widely used for the characterization of DNA (4). This technique is advantageous when compared with classic methods such as chromosomal in situ hybridization or Southern blot, since it provides information on the whole genome without requiring previous knowledge of the genomes under study. In Drosophila, the most widely used technique to detect transposition has traditionally been in situ hybridization on polytene chromosomes, which requires prior knowledge of the genome sequence and TEs (5). This technique requires analysis of a large number of offspring from each hybrid cross and evaluation of many chromosomes. Moreover, the impossibility of analyzing many TEs at one time and the necessity for previous knowledge of chromosomal banding makes this technique difficult to implement. To avoid these drawbacks - especially considering the inexistence of genomic information of many species of Drosophila and insect species in general-we present a protocol with the AFLP technique adapted to detect transposition events in the whole genome of Drosophila. The tool also may be applied to other insect species. Comparisons of AFLP band pattern between hybrid and parental species allow us to rapidly screen the genomic regions subject to instability in hybrid genomes. Hybrid instability can easily be visualized by the presence of new AFLP bands in hybrids that are absent in parents. Each new band can be characterized in order to identify the class of TE responsible for mobilization.

To asses the power of AFLP markers in detecting TEs, we have sequenced a new band (marker TGTCG22) detected in hybrids whose nucleotide sequence is homologous to an exo-endonuclease region of a Helena TE. In situ hybridization of this marker on polytene chromosomes of hybrids and parental species showed new chromosomal bands in hybrids that were not detected in the parental species. Comparison between AFLP and in situ hybridization results allowed us to unequivocally validate the AFLP technique for detecting transposition in hybrids.

\section{Material and methods}

The Drosophila stocks Bu28 and Ko2 are laboratory strains of $D$. buzzatii and D. koepferae species founded from collections of natural populations from Bolivia and Argentina, respectively. Interspecific hybrids were established by crossing two $D$. buzzatii males and one D. koepferae female. Thereafter, three successive mass 


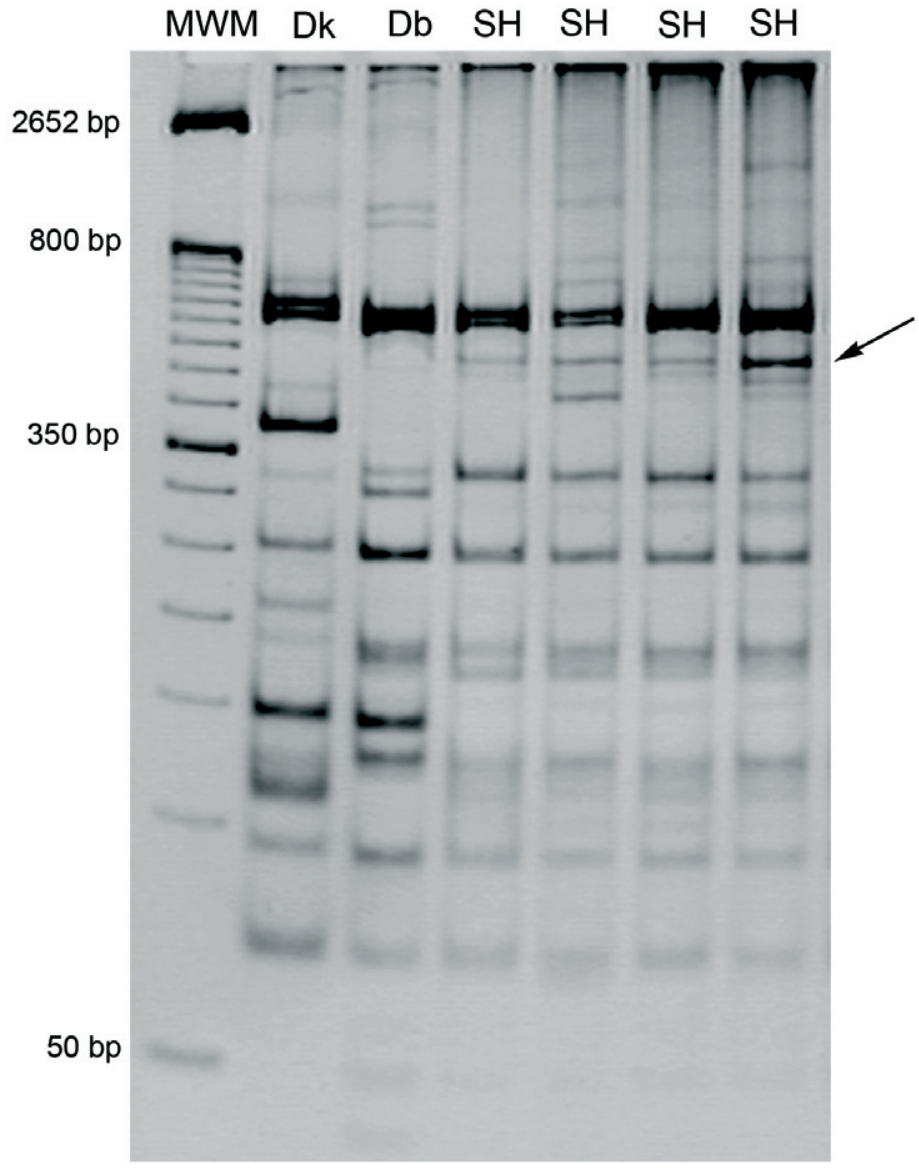

Figure 1. Selective PCR AFLP band patterns using primers with selective nucleotides TG (EcoRI) and TCG (Msel). The arrow indicates the TGTCG22 instability marker band. MWM, molecular weight marker; Dk, D. koepferae; Db, D. buzzatii; SH, segmental hybrids (four hybrids).

backcrosses were performed between F1 hybrid females and $D$. buzzatii males to obtain the corresponding backcross hybrids for each generation $(\mathrm{BC} 1, \mathrm{BC} 2$, and $\mathrm{BC} 3$ ).

Segmental hybrids were also obtained using the backcross protocol. They are characterized by hosting specific chromosome fragments of $D$. koepferae in a D. buzzatii background. These backcross hybrids were selected each generation by the observation of cytological asynapsis in polytene chromosomes in order to preserve the specific introgressed fragments. To illustrate the general validity of this method to assess transposition phenomena in hybrids, we present the results of a particular segmental hybrid which has been introgressed with the region F3-F4 of chromosome 4.

DNA samples of 14 segmental hybrids, 10 third backcross hybrids (BC3), and their parents were isolated by the usual method of individual DNA extraction (6). Simultaneously, DNA samples were extracted from $D$. buzzatii and $D$. koepferae laboratory stocks.
Genomic DNA was digested with EcoRI and $M s e I$ enzymes (New England Biolabs, Ipswich, MA, USA), following the manufacturer's instructions, and linked to adapters EcoRI 5'-CTCGTAGACTGCGTACC-3'; $3^{\prime}$-CATCTGACGCATGGTTAA- ${ }^{\prime}$ and MseI 5'-GACGATGAGTCCTGAG-3'; 3'-CTACTCAGGACTCAT-5' respectively (Sigma-Aldrich, St. Louis, MO, USA). The digestion-ligation reaction was prepared in a total volume of $11 \mu \mathrm{L}$ with $10 \times$ T4 Ligation buffer, $0,5 \mathrm{M} \mathrm{NaCl}$, $100 \times$ BSA, ATP $10 \mathrm{mM}, E c o$ RI Adapter $5 \mu \mathrm{M}, M s e \mathrm{I}$ Adapter $50 \mu \mathrm{M}$, EcoRI $20 \mathrm{U} /$ $\mu \mathrm{L}, M s e \mathrm{I} 10 \mathrm{U} / \mu \mathrm{L}$, T4 DNA Ligase 10 U/ $\mu \mathrm{L}$ (Epicentre, Madison, WI, USA), $\mathrm{H}_{2} \mathrm{O}$, DNA (50 ng/ $\left.\mu \mathrm{L}\right)$. This reaction was incubated overnight at room temperature. Afterward, the total digestion-ligation reaction was diluted to a $200-\mu \mathrm{L}$ final volume.

For preselective PCR reactions, we designed primers by adding one nucleotide (noted in bold) to the previous ones designed for $E c o$ RI and MseI as follows: 5'-GACTGCGTACCAATTCT-3'(EcoRI), 5'-GATGAGTCCTGAG-TAAC-3' (MseI), from Sigma-Aldrich. A PCR preselective reaction was prepared in a total volume of $20 \mu \mathrm{L}$ by adding $10 \times$ PCR buffer, $50 \mathrm{mM}$ $\mathrm{MgCl}_{2}, 5 \mathrm{U}$ DNA EcoTaq polymerase (Ecogen S.R.L., Barcelona, Spain), $2 \mu \mathrm{M}$ dNTPs (Roche, Mannheim, Germany), 2.75 $\mu \mathrm{M}$ EcoRI, $2.75 \mu \mathrm{M} \mathrm{MseI}, \mathrm{H}_{2} \mathrm{O}$, and $5 \mu \mathrm{L}$ DNA digestion-ligation reaction. Amplifications were run in an MJ Research, Inc. thermocycler (Bio-Rad, Hercules, CA, USA) programmed as follows: $2 \mathrm{~min}$ at $72^{\circ} \mathrm{C} ; 20$ cycles of $20 \mathrm{~s}$ at $94^{\circ} \mathrm{C}$ (denaturation), $30 \mathrm{~s}$ at $56^{\circ} \mathrm{C}$ (annealing), $1 \mathrm{~min}$ of extension at $72^{\circ} \mathrm{C}$ then 20 s of final denaturation at $94^{\circ} \mathrm{C} ; 2 \mathrm{~min}$ of final extension at $72^{\circ} \mathrm{C}$; then $30 \mathrm{~min}$ of incubation at $60^{\circ} \mathrm{C}$. The $\mathrm{PCR}$ product was diluted 10 times by adding $\mathrm{H}_{2} \mathrm{O}$ to a final volume of $200 \mu \mathrm{L}$.

For selective PCR reactions, two and three selective nucleotides were added (shown in bold) to EcoRI and $M s e \mathrm{I}$ primers, respectively (for example, EcoRI 5'-GACTGCGTACCAATTCTG-3', MseI 5'-GATGAGTCCTGAGTAATCG-3') and the PCR reaction was performed in a total volume of $20 \mu \mathrm{L}$ including: $10 \times$ PCR buffer, $50 \mathrm{mM} \mathrm{MgCl}$, 5 U DNA EcoTaq polymerase, $2 \mu \mathrm{M}$ dNTPs, $2.75 \mu \mathrm{M}$ EcoRI, $2.75 \mu \mathrm{MMseI}, \mathrm{H}_{2} \mathrm{O}$ and $5 \mu \mathrm{L}$ of DNA from preselective reaction. The thermocycler was programmed as follows: 2 min of initial denaturation at $94^{\circ} \mathrm{C} ; 9$ cycles (reducing $1^{\circ} \mathrm{C}$ each cycle) programmed with $20 \mathrm{~s}$ of denaturation at $94^{\circ} \mathrm{C}, 30 \mathrm{~s}$ of annealing at $66^{\circ} \mathrm{C}$, and $1 \mathrm{~min}$ of extension at $72^{\circ} \mathrm{C} ; 20$ cycles programmed with $20 \mathrm{~s}$ of denaturation at $94^{\circ} \mathrm{C}, 30 \mathrm{~s}$ annealing at $56^{\circ} \mathrm{C}$, and 1 min extension at $72^{\circ} \mathrm{C}$; and finally $20 \mathrm{~s}$ of denaturation at $94^{\circ} \mathrm{C}$ and 30 min incubation at $60^{\circ} \mathrm{C}$

For the AFLP markers, 50 and 32 Eco-Mse AFLP primer combinations were analyzed for BC3 and segmental hybrids, respectively. The AFLP markers were observed in 8\% PAGE gels (AppliChem GmbH, Darmstadt, Germany) with a 29:1 ratio of acrylamide:bisacrylamide concentration. Electrophoresis was performed in TBE $1 \times$ buffer using Protean II xi systems (Bio-Rad). DNA bands were calibrated with the molecular weight marker TrackIt 50-bp Ladder (Invitrogen, Carlsbad, CA, USA) and the samples were subsequently run for $8 \mathrm{~h}$ at $100 \mathrm{~V}$.

To clone the instability AFLP markers, we used the same PCR reagents and concentrations as those used in selective PCR reaction using the following PCR amplification program: 4 min of initial denaturation at $94^{\circ} \mathrm{C} ; 9$ cycles (reducing $1^{\circ} \mathrm{C}$ each cycle) programmed with $20 \mathrm{~s}$ of denaturation at $94^{\circ} \mathrm{C}, 30 \mathrm{~s}$ of annealing at $66^{\circ} \mathrm{C}$, and $1 \mathrm{~min}$ of extension at $72^{\circ} \mathrm{C} ; 20$ cycles programmed with $20 \mathrm{~s}$ of denatur- 

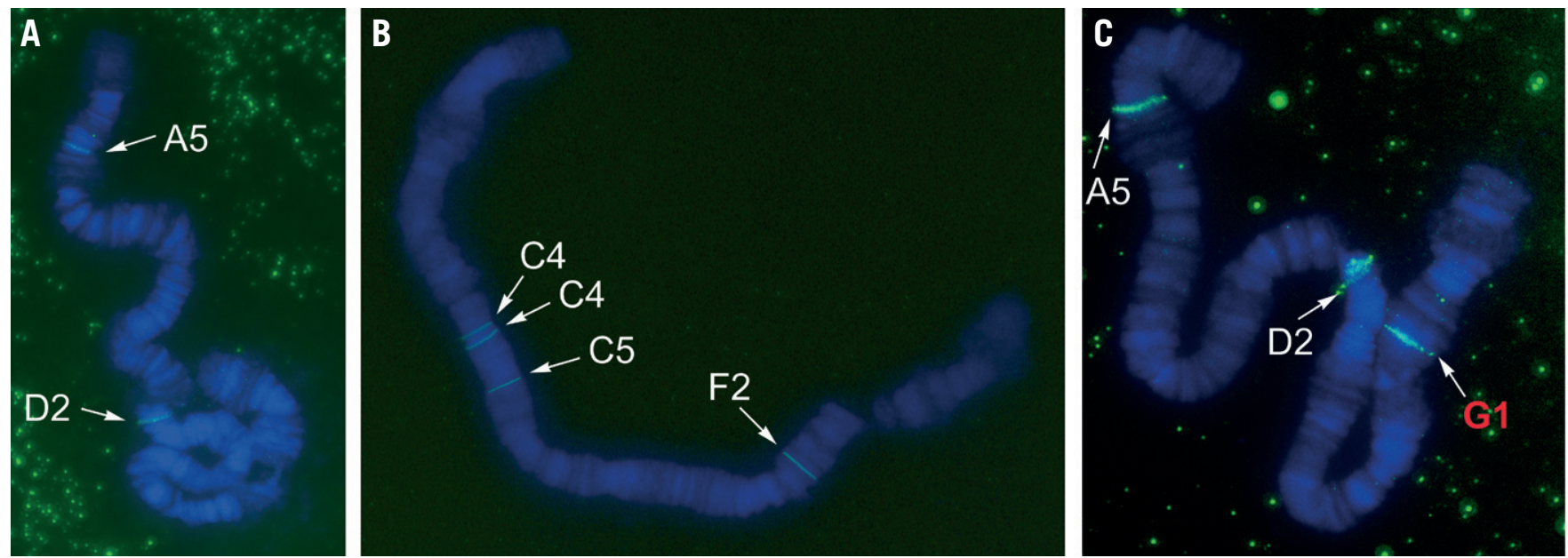

Figure 2. TGTCG22 instability marker in chromosome 5. FISH hybridization of this marker in the parental species D. buzzatii (A), D. koepferae (B), and in the segmental hybrids (C), a new insertion in G1 chromosomal region in the hybrids.

ation at $94^{\circ} \mathrm{C}, 30 \mathrm{~s}$ annealing at $56^{\circ} \mathrm{C}$, and 1 min extension at $72^{\circ} \mathrm{C}$; and finally $20 \mathrm{~s}$ of denaturation at $94^{\circ} \mathrm{C}$ and $30 \mathrm{~min}$ incubation at $60^{\circ} \mathrm{C}$. DNA of the AFLP markers was extracted from the agarose $1 \%$ gel using a kit for DNA gel extraction (Qiagen, Valencia, CA, USA).

Plasmid pGem-T Easy Vector System (Promega, Madison, WI, USA) was used to clone AFLP markers. DH5 $\alpha$ Escherichia coli cells were transformed with each AFLP marker and cultured at $37^{\circ} \mathrm{C}$ overnight in Luria-Bertani (LB) plates containing ampicillin and X-Gal. Positive colonies were cultured in a LB-ampicillin medium, followed by miniprep of the samples. Clones of each AFLP marker were sequenced and their nucleotide sequences analyzed using the alignment tools available in Flybase, NCBI, UCSC and GIRI databases (7-10). In some cases, we found a direct homology with coding or non-coding regions of $D$. mojavensis genome (the closest sequenced species to D. buzzatii and D. koepferae). Repbase, the repetitive DNA database of GIRI, was used to detect homology with transposable elements or repetitive DNA using the forced translated option. The Pfam protein families database (11) allowed the identification of the domains of exo-endonuclease of the Helena element. Complementary information obtained from each database was fundamental in the identification of the TEs present in each AFLP marker checked.

To test the repetitive nature of the resulting hybrid instability markers isolated, we selected the TGTCG22 AFLP instability marker as a probe to hybridize, by FISH, in the polytene chromosomes of segmental hybrids and parental species using the Alexa Fluor 488 Signal Amplification Kit for Fluorescein and Oregon
Green Dye-Conjugated Probes (Cat. no. A-11053; Roche) and DAPI, as described by Invitrogen (http://probes.invitrogen.com/ media/pis/mp11053.pdf).

Polytene chromosomes from salivary glands of segmental $D$. buzzatii-D. koepferae hybrids and parental species, were prepared by the squash method (12). Cytological observations were done using a Zeiss Axio Imager A1 (Jena, Germany). Pictures were captured with an Axio Cam MRc5 photographic camera and visualized with the software Axio Vision ACRel 4.5.

\section{Results and discussion}

AFLPs detect nucleotide changes in the restriction sites of each enzyme detected by the disappearance of some bands (present in parents) or the addition of new bands. We identified three kinds of markers in polyacrylamide gels using this adapted AFLP method for detection of hybrid genome instability: bands from D. buzzatii, bands from $D$. koepferae, and bands originated de novo in the segmental hybrids (instability markers) by transposition (Figure 1). The bands range 50-1000 bp long.

Different AFLP band patterns were observed for each primer combination in BC3 hybrids (50 AFLP primer combinations) and segmental hybrids (32 AFLP primer combinations). Differences were also observed in the number of AFLP genomic bands between hybrids and parental species. The average number of genomic bands observed with Eco-Mse primer combination in BC3 hybrids was: 29.2 bands in hybrids, 21.8 in parental $D$. buzzatii, and 20.8 in maternal D. koepferae. In segmental hybrids, the average number of genomic bands was 18.6 in segmental hybrids, 18.5 in parental D. buzzatii, and
18.2 in maternal D. koepferae. Comparisons between patterns of AFLP bands in hybrids and parental species showed that 9 bands corresponded to instability AFLP markers in BC3 hybrids (detected with 7 AFLP primer combinations) and 11 instability AFLP markers in segmental hybrids (using eight primer combinations). Nucleotide sequences of each AFLP instability marker of $\mathrm{BC} 3$ and segmental hybrids were analyzed in Repbase database. The results showed a high percentage (66-81\%) of instability markers showing direct homology with some TEs; these markers were named AFLP transposition markers. In BC3, segmental hybrids 6 (66\% of hybrid specific markers) and 9 (81\% of hybrid specific markers) AFLP instability markers correspond to TEs. The nucleotide sequences of these 15 AFLP transposition markers correspond to 20 transposable elements belonging to classes I and II. The remaining AFLP instability markers corresponded to coding or noncoding regions of the genome.

In some cases, AFLP transposition markers showed simultaneous homology with two transposable elements. This may be due either to the fact that some markers hold 2 or more elements that move together, or to detection of a unique conserved region common to different TE families.

Bioinformatic analyses of the putative protein coded by the transposition marker TGTCG22 (443 bp) showed homology with the exo-endonuclease region of the Helena TE. FISH hybridization of this marker in polytene chromosomes of segmental hybrids revealed 9 insertions in segmental hybrids, 6 insertions in $D$. buzzatii, and 12 insertions in $D$. koepferae. From the nine euchromatic insertions of the TGTCG22 marker in polytene chromosomes of hybrids, two 
Turning your imaging

systển

visions into

successful

\section{solutions.}

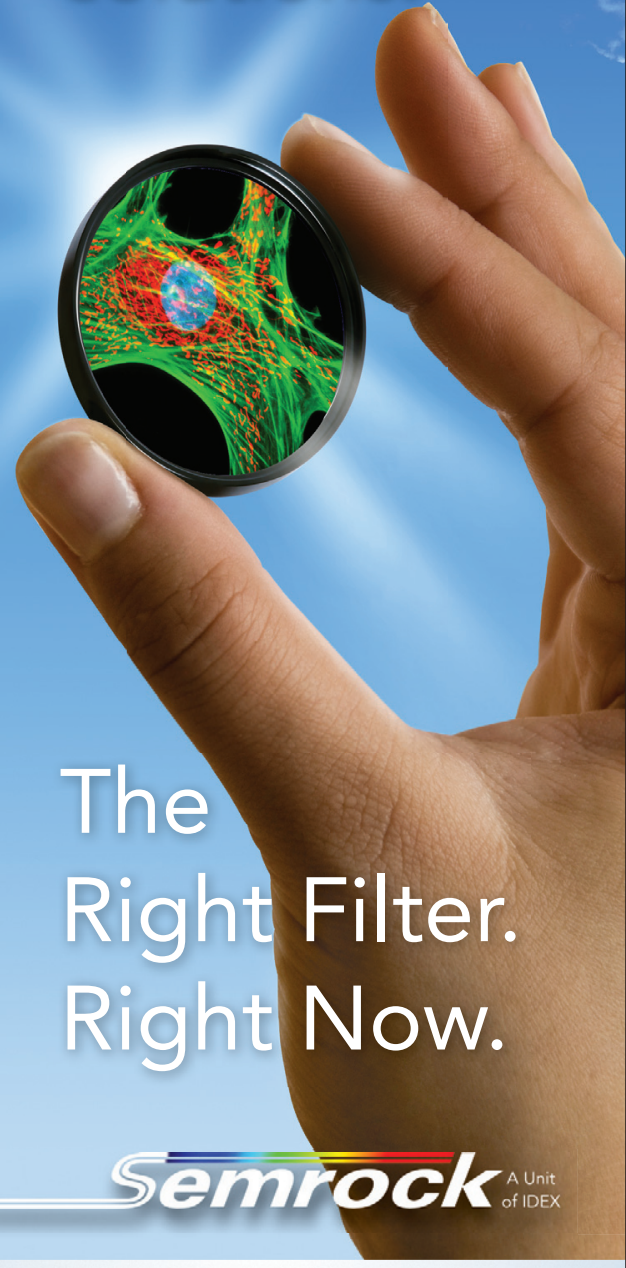

Put the power of our experience
into your hands.

www.semrock.com 1-866-SEMROCK

The Standard in Optical Filters for Life Sciences, Lasers \& Optical Systems
(XA2 and 2E2) were found in both parental species and segmental hybrids and three (3H, 5A5, and 5D2) were shared by $D$. buzzatii and segmental hybrids. The four remaining (XH, 2E3, 3F4, and 5G1) were new insertions (absent from parental species) (Figure 2). These new copies of TGTCG22 marker are exclusive of the hybrid genome that may have originated by transposition of Helena during the hybridization process.

Both the FISH technique on polytene chromosomes and the adapted AFLP protocol presented in this work enable the detection of TE mobilization in genomes $(5,13)$. However, the AFLP protocol coupled with available bioinformatic tools for analyzing and detecting TEs in sequenced genomes (14-16) offer several advantages. First, its application is faster and easier; second, different TEs can be checked simultaneously. Third, results are highly reliable and previous knowledge of chromosomal banding is not required. Moreover, the AFLP technique showed high resolution in detecting genetic instability by transposition. This technique may be expanded to fit the needs of researchers working with other insect species and their hybrids, especially in cases where genomic information is not available Therefore, AFLP markers have demonstrated unequivocally to be an efficient tool for studying TEs in a hybridization processes.

\section{Acknowledgments}

This research was supported by grant nos. POS2003-05904-C02-01, CGL200613423-C02-01, CGL2009-12912-C03-01, and CGL2010-15395 from the Ministerio de Ciencia e Innovación (Spain), and grant nos. 2005SGR 00995 and 2009SGR 636 from Generalitat de Catalunya to the Grup de Biologia Evolutiva. D.V. was supported by a fellowship (PIF) from the Universitat Autonoma de Barcelona (Spain).

\section{Competing interests}

The authors declare no competing interests.

\section{References}

1. Fontdevila, A. 2005. Hybrid genome evolution by transposition. Cytogenet. Genome Res. 110:49-55.

2. Labrador, M., M. Farre, F. Utzet, and A. Fontdevila. 1999. Interspecific hybridization increases transposition rates of Osvaldo. Mol. Biol. Evol. 16:931-937.

3. Michalak, P. 2009. Epigenetic, transposon and small RNA determinants of hybrid dysfunctions. Heredity 102:45-50.
4.Vos, P., R. Hogers, M. Bleeker, M. Reijans, T. van de Lee, M. Hornes, A. Frijters, J. Pot, et al. 1995. AFLP: a new technique for DNA fingerprinting. Nucleic Acids Res. 23:4407-4414.

5. Biémont, C., L. Monti-Dedieu, and F. Lemeunier. 2004. Detection of transposable elements in Drosophila salivary gland polytene chromosomes by in situ hybridization, pp. 21-28. In W.J. Miller and P. Capy (Eds.), Mobile Genetic Elements, Methods in Molecular Biology. Vol. 260. Humana Press, New York.

6. Laayouni, H., M. Santos, and A. Fontdevila. 2000. Toward a Phisical Map of Drosophila buzzatii: Use of ramdomly amplified polymorphic dna polymorphisms and sequence-tagged site landmarks. Genetics 156:1797-1816.

7. Tweedie, S., M. Ashburner, K. Falls, P. Leyland, P. McQuilton, S. Marygold, G. Millburn, D. Osumi-Sutherland, et al. 2009. FlyBase: enhancing Drosophila gene ontology annotations. Nucleic Acids Res. 37:D555D559.

8. Johnson, M., I. Zaretskaya, Y. Raytselis, Y. Merezhuk, S. McGinnis, and T.L. Madden. 2008. NCBI BLAST: a better web interface. Nucleic Acids Res. 36:W5-W9.

9. Kent, W.J. 2002. BLAT - the BLAST-like alignment tool. Genome Res. 12:656-664.

10.Jurka, J., V.V. Kapitonov, A. Pavlicek, P. Klonowski, O. Kohany, and J. Walichiewicz. 2005. Repbase Update, a database of eukaryotic repetitive elements. Cytogenet. Genome Res. 110:462-467.

11.Finn, R.D., J. Mistry, J. Tate, P. Coggill, A. Heger, J.E. Pollington, O.L. Gavin, P. Gunesekaran, et al. 2010. The Pfam protein families database. Nucleic Acids Res. 38:D211-D222.

12. Labrador, M., H. Naveira, and A. Fontdevila. 1990. Genetic mapping of the Adh locus in the Repleta Group of Drosophila by in situ hybridization. J. Hered. 81:83-86.

13. Yu, W., J. Lamb, F. Han, and J. Birchler. 2007. Cytological visualization of DNA transposons and their transposition pattern in somatic cells of maize. Genetics 175:31-39.

14. Quesneville, H., D. Nouaud, and D. Anxolabéhère. 2003. Detection of new transposable element families in Drosophila melanogaster and Anopheles gambiae Genomes. J. Mol. Evol. 57:S50-S59.

15. Andrieu, O., A. Fiston, D. Anxolabéhère, and H. Quesneville. 2004. Detection of transposable elements by their compositional bias. BMC Bioinformatics 5:94.

16. Lerat, E. 2010. Identifying repeats and transposable elements in sequenced genomes: how to find your way through the dense forest of programs. Heredity 104:520-533.

Received 24 January 2011; accepted 23 February 2011.

Address correspondence to María Pilar García Guerreiro, Grupde Biologia Evolutiva. Departament de Genética i Microbiología, Universitat Autonoma de Barcelona, 08193 Bellaterra, Barcelona, Spain. e-mail: mariapilar.garcia.guerreiro@uab.es

To purchase reprints of this article, contact: biotechniques@fosterprinting.com 